\title{
Identification of Dimethoate-containing Water Using Partitioned Dispersive Liquid-liquid Microextraction Coupled with Near-infrared Spectroscopy
}

\author{
Ming ZHANG, Ying GenG, and Bingren XIANG* \\ Center for Instrumental Analysis, Key Laboratory of Drug Quality Control and Pharmacovigilance, \\ Ministry of Education, China Pharmaceutical University, Nanjing 210009, P. R. China
}

(Received January 7, 2011; Accepted March 8, 2011)

\begin{abstract}
A simple, rapid and efficient extraction procedure, partitioned dispersive liquid-liquid microextraction, has been developed in combination with near-infrared spectroscopy for the extraction and discrimination of dimethoate from aqueous samples. For this technique, the appropriate mixture of extraction solvent $\left(\mathrm{CCl}_{4}\right)$ and disperser solvent (THF) was utilized. Partial least squares discriminant analysis was applied to build the model with several pre-process methods over the wavenumber regions between $7100 \mathrm{~cm}^{-1}$ to $7300 \mathrm{~cm}^{-1}$. The best model gave satisfactory classification accuracy, 98.6 $\%$ for calibration set $(n=74)$ and $97.6 \%$ for prediction set $(n=42)$, using preprocessing of standard normal variate followed by Savitzky-Golay first derivative. The method was successfully applied to bottled water, tap water, lake water and farm water samples. The results demonstrated the possibility of near-infrared spectroscopy after partitioned dispersive liquid-liquid microextraction for the identification of water contaminated by dimethoate.
\end{abstract}

Key words—— near-infrared spectroscopy; dimethoate; water analysis; partitioned dispersive liquid-liquid microextraction; partial least squares discriminant analysis

\section{INTRODUTION}

Modern agriculture is supported by the widespread application of pesticides to guarantee productivity and quality. However, the misuse and abuse of these chemicals may lead to risk to environmental damage and human health. Pesticides residues in environment have become a public concern problem. ${ }^{1)}$ Dimethoate (O, O-dimethyl S-methylcarbamoylmethyl phosphoro dithioate) is a broad spectrum contact and systemic organophosphorus insecticide and acaricide used in agriculture and the home garden. Like other organophosphorus, dimethoate is an anticholinesterase which disables cholinesterase, an enzyme essential for the proper functioning of the nervous systems of both humans and insects. ${ }^{2)}$ Dimethoate may spread throughout ecosystems by leaching and runoff from soil into environmental waters because of its high volatility and solubility in water. The reported methods to determine the dimethoate residues are gas chromatography (GC), 3) GC-mass spectrometry (GC-MS),4) liquid chromatography (LC), 5) LCmass spectrometry (LC-MS), 6) enzyme linked immunoassay (ELISA) ${ }^{7)}$ and voltammetry. ${ }^{8)}$

Near-infrared spectroscopy (NIRS) is a fast, eco-

\footnotetext{
*e-mail: cpuxiang@yahoo.com
}

nomic, and widely utilized method which uses the near infrared region of the electromagnetic spectrum (from about $12500 \mathrm{~cm}^{-1}$ to $4000 \mathrm{~cm}^{-1}$ ) .9) It is based on molecular overtone and combination vibrations of $-\mathrm{CH},-\mathrm{OH},-\mathrm{SH}$ and $-\mathrm{NH}$ bonds. Associated with chemometrics, its typical applications include agricultural products, medical sciences, environmental protection and so on. Quality control of pesticide formula, ${ }^{10)}$ detection for pesticide residues in soil ${ }^{11,12)}$ and agriculture products ${ }^{13-15)}$ have been successfully performed by NIRS.

In aquatic environmental samples the residues are often present only at trace concentrations, whose signals are almost lost in the strong near-infrared absorption of water. The sufficient sensitivity can not be reached. Therefore, the pesticides residues are needed to extracted and enriched into organic solvents by sample preparation.

The most widely extraction techniques used for many years in environmental analysis are liquid-liquid extraction (LLE) ${ }^{16,17)}$ and solid-phase extraction (SPE) . ${ }^{18,19)}$ LLE is tedious, time-consuming and normally requires large amounts of organic solvents which are environmental unfriendly. Although SPE reduces the use of organic solvent, it can be relatively expensive. Various modes of liquid-phase microextraction (LPME) have emerged since the middle-to- 
late 1990s such as single drop microextraction (SDME) ${ }^{20)}$ hollow-fiber liquid-phase microextraction (HF-LPME), 21) liquid-liquid-liquid microextraction (LLLME), ${ }^{22)}$ wetting film extraction (WFE) ${ }^{23)}$ and cloud point extraction (CPE) .24) LPME generally miniatures the environmentally sound sample preparation procedures but do not reduce long extraction time.

Dispersive liquid-liquid microextraction (DLLME) ${ }^{25)}$ is based on a ternary component solvent system. An appropriate mixture of water-immiscible organic extractant and water-miscible dispersive solvent is injected into aqueous samples by syringe to form small droplets. After the formation of cloudy solution, the surface area between the extracting solvent and the aqueous sample becomes very large, so the extracting time is very short. ${ }^{26)}$ Besides time-saving, the advantages of DLLME method are simplicity of operation, low-cost, high recovery and enrichment factor. ${ }^{25)}$ It is observed that at higher dispersive solvent volumes, the solubility of the analytes increases lowering analytes partition with extractant droplets leading to a decrease in efficiency, especially when extracting a high polar chemical. The above limitation was addressed by a DLLME configuration termed partitioned dispersive liquid-liquid microextraction (PDLLME). In PDLLME a dispersive solvent that was also partitioned in the extractant droplets was chosen, Due to the polar nature of the dispersive solvent and based on the partition coefficient of the polar compounds in a dispersive solvent, the analytes were extracted into the extractant droplets as well as dispersive solvent, which led to the increase in extraction efficiency. ${ }^{27,28)}$

In this work, the feasibility of detecting dimethoate in environmental waters based on NIRS combined with partial least square discriminant analysis (PLSDA) was investigated. PLLME was firstly employed as the sample preparation technique, which extracted semi-polar dimethoate into organic solvents to avoid interference signals due to water and increase concentration. The timesaving technique made sure the presentation and analysis of a sample could be accomplished rapidly with promising performance. The possible application of the method in real water samples was also investigated.

\section{MATERIALS AND METHODS}

Standards and Samples Dimethoate (97\% purity) was purchased from Institute for the Control of Agrochemicals, Ministry of Agriculture (ICAMA), Methanol (Jiangsu Hanbon Science \& Technologies Co. Ltd., China), tetrachloromethane $\left(\mathrm{CCl}_{4}\right.$, Shanghai Experimental Reagent Co. Ltd., China), hexyl hydride (Nanjing Chemical Reagent Co. Ltd., China) and tetrahydrofuran (THF, Shanghai Lingfeng Chemical Reagent Co. Ltd., China) were HPLC grade. Sodium chloride (Nanjing Chemical Reagent Co. Ltd., China) was used to adjust the ionic strength of the samples. Double-distilled water was employed for the preparation of aqueous solutions. Bottled water (Guangdong Robust Co. Ltd., China) was obtained from a local supermarket. Tap water, farm water and lake water were collected from our laboratory, the local area of Nanjing and the Xuanwu Lake (Nanjing, China), respectively. All the samples were filtered through $0.45 \mu \mathrm{m}$ filter twice prior to PDLLME procedure.

Samples Preparation Stock solution of dimethoate $\left(100 \mu \mathrm{g} \mathrm{ml}^{-1}\right)$ was prepared in double-distilled water. Among prepared samples, dimethoate-free samples were blank double-distilled water, and the dimethoate-containing samples were prepared by diluting different amounts of the standard stock solution with double-distilled water to the varying concentrations.

The partitioned dispersive liquid-liquid microextraction procedure was as follows: A $6 \mathrm{ml}$ water sample solution containing the target analyte prepared according to the procedures described above was placed in a $10 \mathrm{ml}$ glass centrifuge tube with a conical bottom. One $\mathrm{g}$ of sodium chloride was added to the glass tube and dissolved completely. Three hundred $\mu \mathrm{l}$ of mixed organic solvent $\left(\mathrm{CCl}_{4}: \mathrm{THF}=1: 2, \mathrm{v} / \mathrm{v}\right)$ was added into the centrifuge tube using a syringe, and then the tube was shaken violently for $60 \mathrm{~s}$. A cloudy solution (water, tetrachloromethane, and tetrahydrofuran) was formed in the test tube which was stable for a long time. The extracted dimethoate in the mixed phase of $\mathrm{CCl}_{4}$ droplets and $\mathrm{CCl}_{4}$-partitioned THF were collected by centrifugation $(4000 \mathrm{rpm}, 5 \mathrm{~min})$ and $160 \mu \mathrm{l}$ of the sediment organic phase was transferred into a microsyringe for subsequent analysis. A hundred thirty $\mu$ l of sediment phase was used for recording NIR spectra. The remaining $30 \mu \mathrm{l}$ was 
evaporated to dryness under a stream of nitrogen. Because the ECD is sensitive to extractant $\mathrm{CCl}_{4}$ at or below 2 parts per billion, the residue was reconstituted in a $10 \mu \mathrm{l}$ hexyl hydride. An aliquot of $2 \mu \mathrm{l}$ was injected into GC-ECD.

GC Analysis The reference method for the determination of dimethoate was performed with a Shimadzu GC-14B gas chromatograph (Tokyo, Japan) equipped with an electron capture detector (ECD). A personal computer equipped with the N2000 chromatography data system (Zhejiang University Star Instrument Technology Co., Ltd.) was used for data processing. Separation was carried out on a HP-1, $30 \mathrm{~m} \times 0.22 \mathrm{~mm}$ capillary column (Agilent Technologies, Santa Clara, USA) with a $0.25 \mu \mathrm{m}$ stationary film thickness. Ultra pure nitrogen was adopted as the carrier gas at constant flow rate of

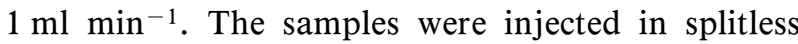
mode. The injection port was held at $200^{\circ} \mathrm{C}$. The oven and ECD temperature was maintained at $180^{\circ} \mathrm{C}$ and $210^{\circ} \mathrm{C}$ respectively.

NIR Methods A multipurpose analyzer (MPA) FT-NIR spectrometer from Bruker Optics (Ettlingen, Germany), equipped with a quartz beamspliter, an air cooled NIR source and an InGaAs detector, was used in this study. For instrument control and data acquisition the OPUS (Optics User Software) Version 5.5 from Bruker was employed. Each individual spectrum was the average of 32 scans collected with a resolution of $4 \mathrm{~cm}^{-1}$ over the wavenumber range of $12500-4000 \mathrm{~cm}^{-1}$ in transmission mode. Triplicate spectra were acquired for each sample at the temperature of $25^{\circ} \mathrm{C}$. The mean of three spectra of the same sample was used in subsequent spectral analysis steps.

Chemometric analysis, including spectra preprocess and qualitative determination of dimethoate was performed in MATLAB 7.5 (Math Works Inc. Natick, USA) with PLS Toolbox 5.8 (downloaded from http://www.eigenvector.com/software/pls toolbox.htm.)

Partial least squares regression is a multivariate linear projection method, which used to find the fundamental relations between two matrices ( $\mathbf{X}$ and $\mathbf{Y}$ ), i.e., a latent variable (LV) approach to modeling the covariance structures in these two spaces. A PLS model will try to find the multidimensional direction in the $\mathbf{X}$ space that explains the maximum multidimensional variance direction in the $\mathbf{Y}$ space. PLS decomposes the $(n \times N)$ matrix of zero-mean varia- bles $\mathrm{X}$ and the $(\mathrm{n} \times \mathrm{M})$ matrix of zero-mean variables $\mathrm{Y}$ into the form:

$$
\begin{aligned}
& \mathrm{X}=\mathrm{TP}^{\mathrm{T}}+\mathrm{E} \\
& \mathrm{Y}=\mathrm{UQ}^{\mathrm{T}}+\mathrm{F}
\end{aligned}
$$

Where the $\mathbf{T}, \mathbf{U}$ are $(n \times p)$ matrices of the $p$ extracted score vectors, the $(\mathrm{N} \times \mathrm{p})$ matrix $\mathbf{P}$ and the $(\mathrm{M} \times \mathrm{p})$ matrix $\mathbf{Q}$ represent matrices of loadings and the $(n \times N)$ matrix $\mathbf{E}$ and the $(n \times M)$ matrix $\mathbf{F}$ are the matrices of residuals. The $\mathbf{T}$ and $\mathbf{U}$ represent response information after removing most noise. Based on the correlation between them, a regression model was formed:

$$
\mathrm{U}=\mathrm{TB}
$$

Partial least squares discriminant analysis (PLSDA) is a classification method based on modelling the differences between several classes with PLS. If there are only two classes to separate, the PLS model uses one dummy variable, which codes for class membership as follows: -1 for members of Class $\mathrm{A},+1$ for members of Class B (in this study, -1 for water samples not containing dimethoate and +1 for water samples containing dimethoate). A discriminant model was developed by regression of the spectral data (X) against the assigned dummy variable $(\mathbf{Y})$. The model based on experimental data was established in order to assign unknown samples to a previously defined sample class based on pattern of its measured features. Then a leave-one-out cross-validation was carried out. In the validation, all samples in calibration set except one were used to construct model and then the model was used to predict the remained sample. The procedure was repeated, leaving out each of the samples of the calibration set in turn. The threshold was set to 0 , and a sample was considered to be categorized correctly if the predicted value lay on the same side of the midpoint of the assigned value. A sample was identified as Class A if its predicted value was below 0 and as Class B if its predicted value was above 0 . The number of PLSDA factors was selected according to the lowest rootmean-square-error (RMSECV) of cross validation. The prediction ability of the established models was assessed by classification accuracy of calibration set and prediction set. It is commonly agreed that the higher the correct classification rate, the better the model.

\section{RESULTS AND DISCUSSION}

GC Performances The GC reference method 
confirmed that all the blank samples were dimethoatefree and the other samples did contain dimethoate with the concentrations in the range of $0.04 \mu \mathrm{g} \mathrm{ml}^{-1}$ to $0.30 \mu \mathrm{g} \mathrm{ml}^{-1}$.

Selecting of Calibration Set and Prediction Set

All 116 samples were divided into two subsets. The calibration set, which was used to build the model, had 74 samples including 27 dimethoate-free samples and 47 samples containing dimethoate. The prediction set was formed by the remaining 42 samples with 18 blank samples and 24 contaminated samples. The experiments were carried out in 4 different days considering the systematic error arising from weather condition and instrumental instability.

Characteristics and Pre-processing of Spectra

Shapes of the raw spectra of all samples were quite homogeneous and it is difficult to find specific band to discriminate whether dimethoate was included in the aqueous samples since NIR bands are severely overlapped due to overtones and combination modes.

Different spectra pre-processing were applied to enhance the signal-to-noise ratio and spectral features, including Savitzky-Golay (SG) first and second derivative, and standard normal variate transformation (SNV).

First and second derivatives eliminate baseline drifts and enhance small spectral differences. To avoid Derivative enhancement of noise, spectra derivation is done by using the Savitzky-Golay algorithm, which is a moving window averaging method: a window is selected where the data are fitted by a poly- nomial of a certain degree. The central point in the window is replaced by the value of the polynomial.

$\mathrm{SNV}$ is a mathematical transformation of the spectra by calculation of the standard normal variation at each wavelength removing slope variation on an individual sample basis by the use of the following calculation:

$$
A_{i(S N V)}=\frac{A_{i, k}-\overline{A_{i}}}{\sqrt{\frac{\sum_{k=1}^{m}\left(A_{i, k}-\overline{A_{i}}\right)^{2}}{m-1}}}
$$

Where $\overline{A_{i}}$ is the mean of the individual spectrum from each value $A_{i, k}, k$ is the number of wavenumbers, and $i$ is the number of spectra.

The result of Savizky-Golay first derivative with smoothing over 15 points was shown in Fig. 1, with the region between $7100 \mathrm{~cm}^{-1}$ and $7300 \mathrm{~cm}^{-1}$ enlarged, where the key differences were observed. The absorption bands in the range of $7300 \mathrm{~cm}^{-1}-7100$ $\mathrm{cm}^{-1}$ may be ascribed to the first overtone of secondary amides (- $\mathrm{CONH}-)$ and the combination of the stretching and bending of methyl group $\left(\mathrm{CH}^{-}\right)$of dimethoate. To enhance the performance of classification and decrease the computation time, only this wavelength region combined with different data preprocessing was used in following investigation.

Partial Least Squares Discriminant Analysis In the study PLSDA was used to performance classification. Water samples containing dimethoate were assigned a dummy value 1 and those not containing dimethoate were -1 . Table 1 showed the statistics

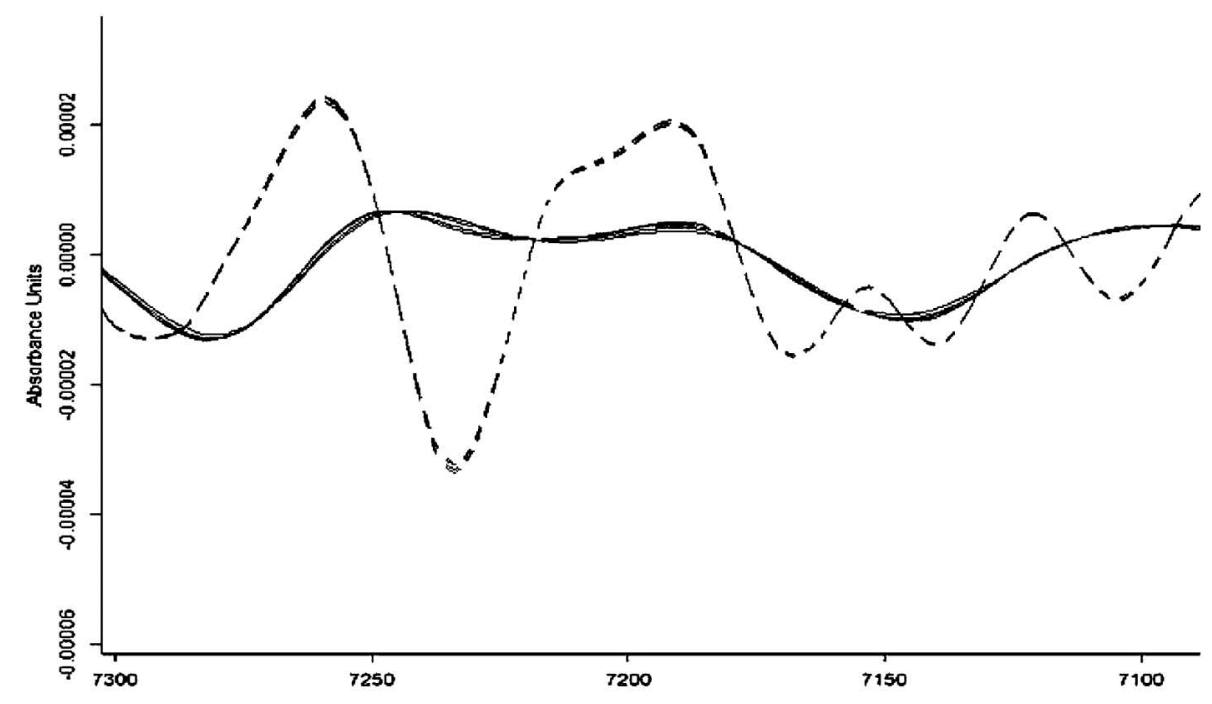

Fig. 1. Savitzky-Golay First Derivative Spectra of Sediment Phase 7100-7300 $\mathrm{cm}^{-1}$, Solid line: dimethoate-free samples, Dotted line: dimethoate-containing samples. 
for the number of incorrectly classified samples with MSC, SNV and derivative spectra pre-process using region from 7300 to $7100 \mathrm{~cm}^{-1}$. The results indicated that all kinds of pre-process can improve accuracy compared with raw spectra. The optimum model was obtained by the use of spectral data after SNV and first derivative data pre-process step with PLS factors of 5. It is known that the side effects of derivatives on spectroscopic data are the loss of the original shape of the spectral curve and the reduction of the signal-tonoise ratio. The reason why SNV + SG 1st derivative method is more excellent than SNV + SG 2ed derivative method may be related to those side effects, which increase as the derivative order goes higher. The predicted values of samples in calibration set and

Table 1. The Results of Discrimination Models Developed by PLSDA Using Varying Data Pre-process

\begin{tabular}{ccccc}
\hline \hline $\begin{array}{c}\text { Data } \\
\text { pre-process }\end{array}$ & Factors & \multicolumn{2}{c}{$\begin{array}{c}\text { No. of incorrectly } \\
\text { classified samples } \\
\text { Calibration Prediction } \\
\text { set }(n=74)\end{array}$} & $\begin{array}{c}\text { Correct } \\
\text { percent } \\
(\%)\end{array}$ \\
\hline set $(n=42)$ & \\
\hline None & 3 & 8 & 22 & $73.20 \%$ \\
SG 1st derivative & 3 & 6 & 6 & $90.20 \%$ \\
SG 2ed derivative & 4 & 2 & 3 & $92.90 \%$ \\
$\begin{array}{c}\text { SNV + SG 1st } \\
\text { derivative }\end{array}$ & 5 & 1 & 1 & $98.50 \%$ \\
$\begin{array}{c}\text { SNV+SG 2ed } \\
\text { derivative }\end{array}$ & 5 & 1 & 3 & $96.40 \%$ \\
\hline
\end{tabular}

predication set based on the optimum model were shown in Figs. 2 and 3, respectively. The threshold was set to 0 for detecting whether water samples containing dimethoate. The sample containing dimethoate was classified correctly if the value was above 0 , and the dimethoate-free sample was classified correctly if the value was below 0 . Only one sample of calibration set and one sample in prediction set were misjudged. The correct classification rates were 98.6 $\%$ for calibration set, $97.6 \%$ for prediction set and $98.2 \%$ for all samples. It is known that the lowest concentration of dimethoate for samples is $0.04 \mu \mathrm{g}$ $\mathrm{ml}^{-1}$ from GC results and a satisfactory correct classification rate $(>98 \%)$ was obtained. So the limit of detection (LOD) of the qualitative analysis method was $0.04 \mu \mathrm{g} \mathrm{ml}^{-1}$.

Application of the Technique To demonstrate the capability of the proposed technique, the procedure was applied to the analysis of dimethoate in bottled water, tap water, lake water and farm water samples. Each kind of water included 5 samples. The prediction values of all 20 samples were below 0 , which indicated no dimethoate was found in the actual water. The same results were obtained by GC method. It suggested the method was potential to accurately predict whether dimethoate was contained in environmental water samples.

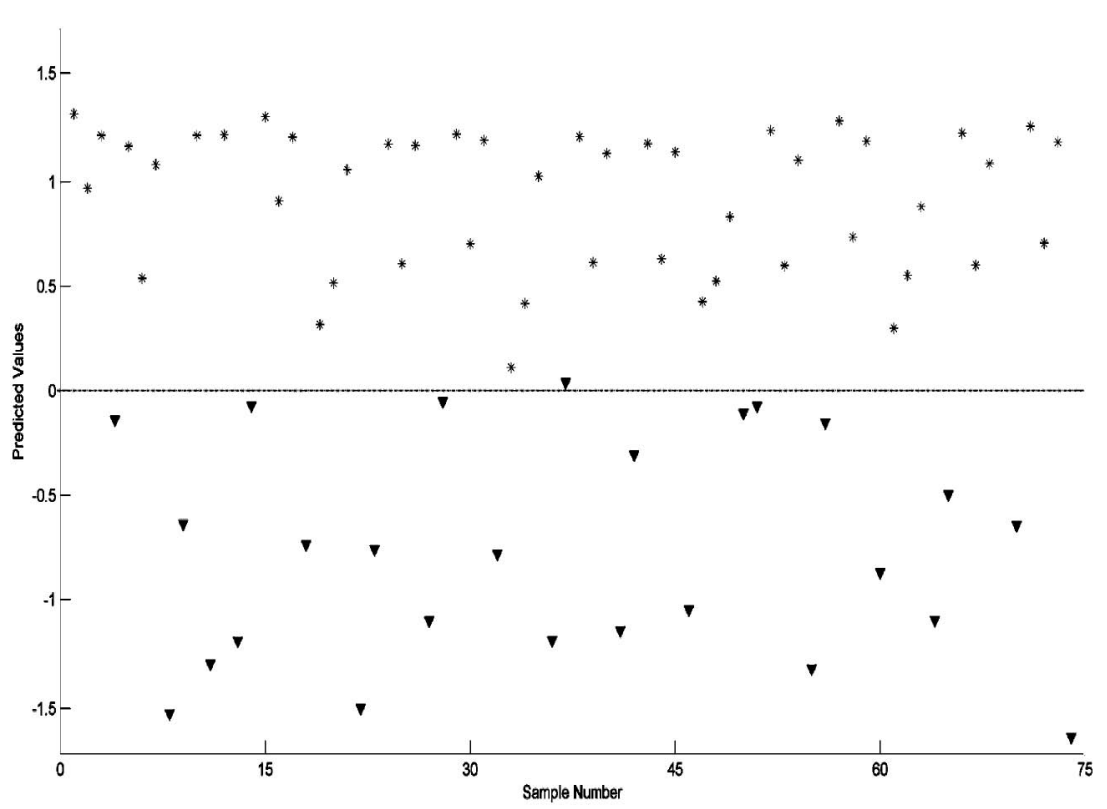

Fig. 2. The Predicted Results of PLSDA in Calibration Set " $\boldsymbol{\nabla}$ ", dimethoate-free samples, " $*$ ", dimethoate-containing samples. 


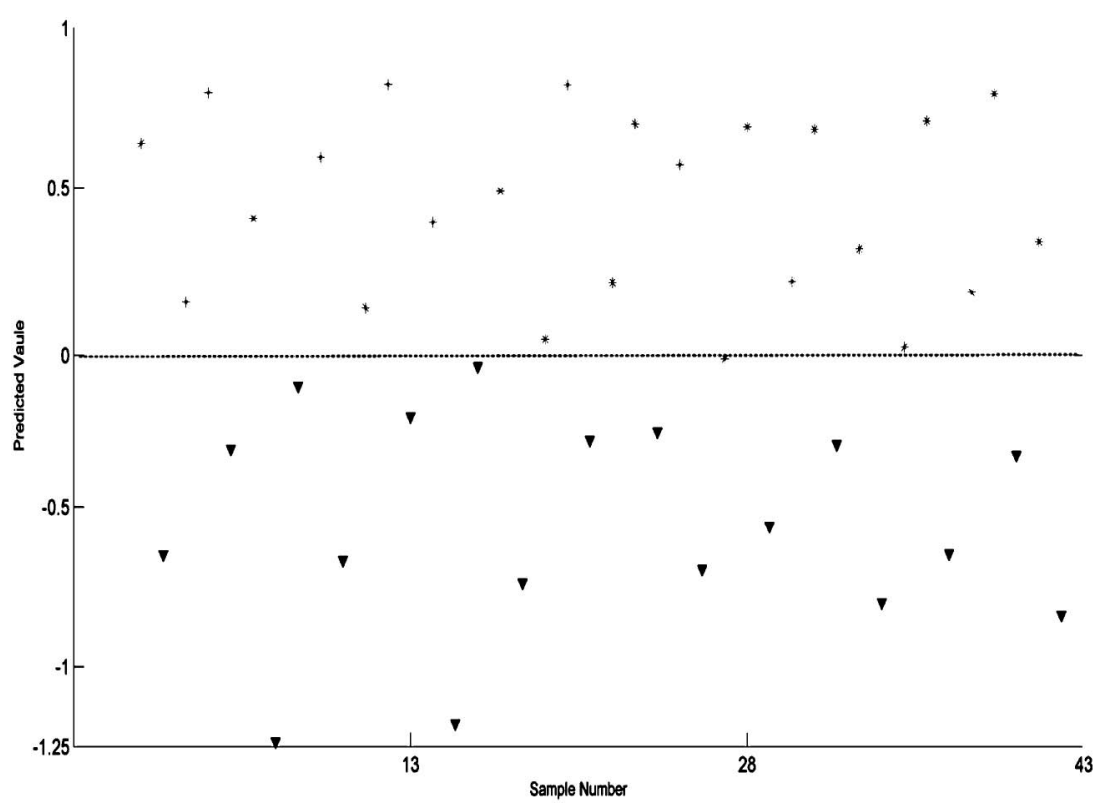

Fig. 3. The Predicted Results of PLSDA in Prediction Set

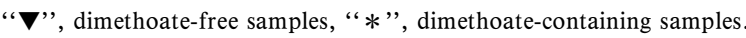

\section{CONCLISION}

In this study, NIRS combined with PLSDA were preliminarily investigated for the identification of water samples contaminated by dimethoate after employing PDLLME as the sample preparation technique. The results of this study show that a satisfactory correct classification rate can be obtained after spectral data region and pre-process optimization. The method which can be finished in less than 15 minutes compared to 30 min employing GC provides a speedy, low-cost, high automatization and easy-touse alternative to the traditional reference procedure. The study results are encouraging for development of other application of liquid-phase microextraction in NIRS.

Acknowledgment Finacial supports from the CEEUSRO combination projects of Department of Education of Guangdong Province ( No. 2007A09032100) are gratefully acknowledged.

\section{REFERENCES}

1) Ritter L., Goushleff N., Arbuckle T., Cole D., Raizenne M., J. Toxicol. Env. Heal. B, 9, 441 -456 (2006).

2) John H., Eddleston M., Clutton R. E., Worek F., Thiermann H., J. Chromatogr. B, 878, 1234-1245 (2010).
3) Xi Y., Dong H., Anal. Sci., 23, 295-298 (2007).

4) Koesukwiwat U., Lehotay S. J., Miao S., Leepipatpiboon N., J. Chromatogr. A, 1217, 6692-6703 (2010).

5) Ali B., Farid A., Rafique M., Shah H., Khattak A., Food Chem., 114, 286-288 (2009).

6) Baugros J. B., Giroud B., Dessalces G., Grenier-Loustalot M. F., Cren-Olivé C., Anal. Chim. Acta, 607, 191-203 (2008).

7) Brena B. M., Arellano L., Rufo C., Last M. S., Montaño J., Cerni E. E., Gonzalez-Sapienza G., Last J. A., Environ. Sci. Technol., 39, 3896-3903 (2005).

8) Díaz T., Guiberteau A., Soto M., Electroanalysis, 10, 695-702 (2006).

9) Rao Y. L., Xiang B. R., Yakugaku Zasshi, 129, 881-886 (2009).

10) Armenta S., Garrigues S., de la Guardia M., Vib. Spectrosc., 44, 273-278 (2007).

11) Bengtsson S., Berglöf T., Kylin H., B. Environ. Contam. Tox., 78, 295-298 (2007).

12) Brunet D., Woignier T., Lesueur-Jannoyer M., Achard R., Rangon L., Barthès B. G., Environ. Pollut., 157, 3120-3125 (2009).

13) Zhou Y., Xiang B. R., Wang Z., Chen C. Y., Anal. Lett., 42, 1518-1526 (2009).

14) Sánchez M., Flores-Rojas K., Guerrero J. E., Garrido-Varo A., Pérez-Marín D., Pest. Ma- 
nag. Sci., 66, 580-586 (2010).

15) Fen D., Hong T., Zhang K., Hong Y., Intelligent Computation Technology and Automation, International Conference on 2010, 781783 (2010).

16) Tan G. Analyst, 117, 1129-1132 (1992).

17) Yasuhara A., Shiraishi H., Nishikawa M., Yamamoto T., Uehiro T., Nakasugi O., Okumura T., Kenmotsu K., Fukui H., Nagase M., Ono Y., Kawagoshi Y., Baba K., Noma Y., J. Chromatogr. A, 774, 321-332 (1997).

18) Steen R., Hogenboom A. C., Leonards P., Peerboom R., Cofino W., Brinkman U. A., J. Chromatogr. A, 857, 157-166 (1999).

19) Jeannot R., Sabik H., Sauvard E., Dagnac T., Dohrendorf K., J. Chromatogr. A, 974,143159 (2002).

20) Saraji M., Esteki N, Anal. Bioanal. Chem.,
391, 1091-1100 (2008).

21) Tahmasebi E., Yamini Y., Saleh A. Analysis, 877, 1923-1929 (2009).

22) Bagheri H., Khalilian F., Ahangar L. E., J. Sep. Sci., 31, 3212-3217 (2008).

23) Adam I., Anthemidis A. N., Talanta, 77, 1160 -1164 (2009).

24) Yetimoğlu E., Urucu O., Gündüz Z. Y., Filik H., Anal. Lett., 43, 1846-1856 (2010).

25) Rezaee M., Assadi Y., Milani Hosseini M. R., Aghaee E., Ahmadi F., Berijani S., J. Chromatogr. A, 1116, 1-9 (2006).

26) Rezaee M, Yamini Y., Faraji M., J. Chromatogr. A, 1217, 2342-2357 (2010).

27) Melwanki M. B., Fuh M., J. Chromatogr. A, 1207, 24-28 (2008).

28) Chou T., Lin S., Fuh M., Talanta, 80, 493498 (2009). 